\title{
A Conservative Macroscopic Model for Binary-mixture Fluidized Beds
}

\author{
Mohamed Sobhi Alagha1,2, Pal Szentannai \\ ${ }^{1}$ Department of Mechanical Engineering, Faculty of Engineering, Kafrelsheikh University, 33516 Kafrelsheikh, El-Giesh Street 5, \\ Egypt \\ 2 Department of Energy Engineering, Faculty of Mechanical Engineering, Budapest University of Technology and Economics, \\ H-1111 Budapest, 9 Műegyetem rkp., Hungary \\ * Corresponding author, e-mail: szentannai@energia.bme.hu
}

Received: 28 October 2020, Accepted: 04 March 2021, Published online: 03 August 2021

\begin{abstract}
Two approaches are commonly used for modeling the vertical mixing of binary-mixture fluidized beds, Computational Fluid Dynamics (CFD) and macroscopic modeling. A common realization of the latter one is the Gibiralo-Rowe (G-R) model, which uses the Two-Phase Theory. This macroscopic model obviously overperforms CFDs regarding computational cost; however, determining its coefficients is a still challenging issue. Although several methods were published for solving this, the general problem with most of them remains their neglecting the conservation of mass. In the present new procedure, the mass conservation is applied to correct the values of the G-R model coefficients estimated from known equations. The present model was validated on a wide variety of fluidized bed systems. The results show that this conservative and macroscopic model gives more accurate predictions than the recently published other macroscopic models, and this one is, in general, better than the CFD model from the perspective of prediction accuracy as well.

\section{Keywords}

fluidized beds, mixing, segregation, macroscopic modeling, CFD, validation
\end{abstract}

\section{Introduction}

Nowadays, gas-solid fluidized beds are used in many industrial and power generation applications due to their excellent mixing and heat transfer characteristics. The binary-mixture fluidized bed contains a mixture of two particulate species of different sizes and densities. For process optimization and development mixing of this non-homogeneous system needs more understanding.

Modeling is an essential tool that provides information for studying and analyzing a given phenomenon. However, modeling of this multi-phase interactive structure is a challenging problem that even the complex-algorithm Computational Fluid Dynamics (CFD) models do not give the exact solution $[1,2]$. The evolution of these models is running continuously to touch mesoscopic aspects related to the complex nature of the real phenomenon [3-5]. However, the CFD models still require a long time and a high computation cost to carry out a comprehensive validation with various experimental cases. On the other hand, some macroscopic models, such as the
Gibilaro-Rowe (G-R) model [6] can give good predictions of the segregation profile if a suitable set of coefficients is selected $[7,8]$.

Some previous studies have attempted to find a generally valid form of the G-R model [8-11]. Among them, only the Turrado et al. [8] version is one of the results of satisfying the mass conservation of the binary-mixture bed components. However, similarly to the majority of published applications, Turrado et al. [8] used a simplified version of the G-R model. This analytically solvable form disregards one of the four main mechanisms covered by the full G-R model, hence the simplification results in limiting the G-R model applicability [12]. Recently, Girimonte et al. [13] proposed an alternative macroscopic method that uses a fitting parameter that depends on the final fluidization velocity [14].

In the present study, the differential equations of the G-R mixing/segregation theory are solved numerically to include all the mixing/segregation mechanisms. Moreover, in the procedure proposed by us, the mass conservation 
framework is applied to correct the values of the model coefficients that are estimated from the most cited equations published by others. Then, the segregation profile has to be corrected by factorizing the model coefficients, and the proposed procedure also includes a new segregation layer formula for linking the G-R model calculation with the fluidization ratio. This latter formula is tested, and furthermore, the model itself is evaluated using various experimental cases given from the literature. Finally, a detailed systematic comparison with other recently published macroscopic models is presented.

\section{Theory}

Earlier, Toomey and Johnstone [15] proposed the TwoPhase Theory (TPT) to describe the movement of the solids in the fluidized bed by the interaction between the bulk and wake phases. In the binary-mixture fluidized beds, the particulate system is composed of two-particle species of different densities and sizes. The particle species which sinks to the bed bottom is called jetsam, while the particle species which tends to float towards the bed top is called flotsam [16]. Gibilaro and Rowe [6] used the TPT principle to analyze the jetsam movement in the bulk and wake phases in the binary-mixture fluidized beds, as shown in Fig. 1.

The complete G-R model is given by Eqs. (1)-(4).

$$
\begin{aligned}
& \beta \frac{\partial^{2} C_{B}}{\partial Z^{2}}+\left(\lambda+1-2 C_{B}\right) \frac{\partial C_{B}}{\partial Z}+\gamma \lambda\left(C_{W}-C_{B}\right)=0, \\
& \lambda \frac{\partial C_{W}}{\partial Z}-\gamma \lambda\left(C_{B}-C_{W}\right)=0,
\end{aligned}
$$

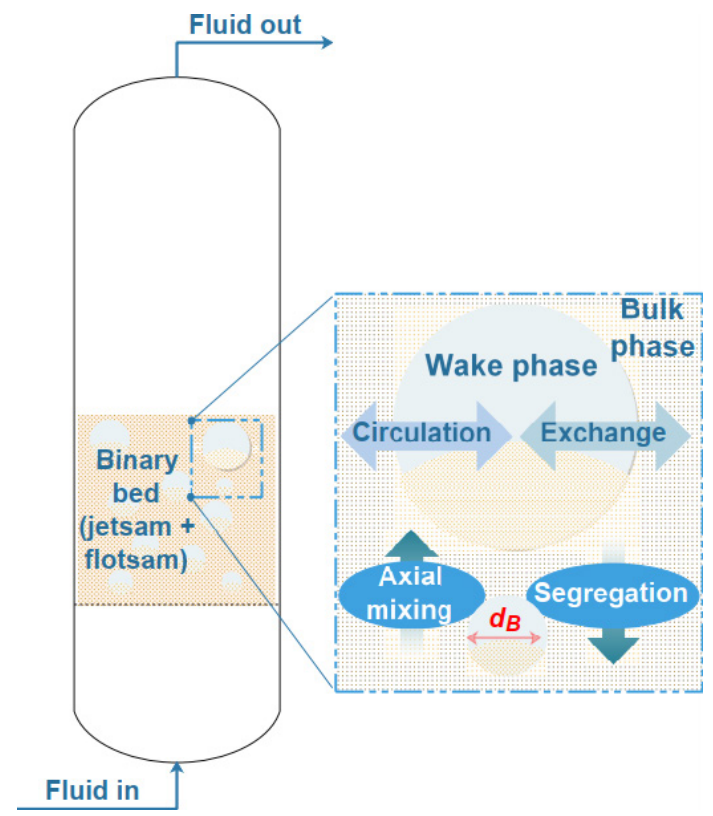

Fig. 1 A schematic illustration of the G-R model mechanisms

$$
\begin{gathered}
C_{\text {ave }}=\left(1-f_{W}\right) C_{B}+f_{W} C_{W}, \\
X_{\text {ave }}=\frac{C_{\text {ave }}\left(\frac{\rho_{j}}{\rho_{f}}\right)}{1-\left(1-\frac{\rho_{j}}{\rho_{f}}\right) C_{\text {ave }}},
\end{gathered}
$$

where $C_{B}$ and $C_{W}$ are the bulk phase and wake phase volume fractions of jetsam concentration $C_{a v e}$ at a given height, while $\beta, \lambda, \gamma$, and $f_{W}$ are the G-R model coefficients. These coefficients are the most critical components of the G-R model. For example, Fig. 2 shows the variation of the jetsam axial concentration profile estimated from the G-R model by using different coefficient values. Accordingly, the model prediction is very sensitive to the values of these coefficients. However, the big challenge since time is finding a generally valid set of coefficients of the G-R model.

The direct linkage of the segregation model coefficients to the system's physical properties requires a huge number of experiments on different systems at different operating conditions. Instead, we relied on the most cited G-R model coefficients' equations available in the literature (see Table 1) [17-20] to reflect the relationship with the physical conditions. These equations are based on bubble-bed fluid dynamics; thus, they can give a general solution. And accordingly, the physical conditions of the system are implemented as inputs to the present conservative G-R model, as shown in the calculation flow chart in Fig. 3. We used in the present study the bubble size correlation of Horio and Nonaka [20], however other bubble size correlations were used in the literature [21, 22]. The reason is that the Horio and Nonaka [20] bubble size correlation does not depend on the bed height.

Fig. 3 shows the flowchart of the standard calculation of the jetsam axial concentration profile for the G-R model. Here the G-R model coefficients are estimated from the input physical properties of the binary-mixture system (Step A) by implementing the set of equations in Table 1 (Step B). Then, the second-order differential equation of the G-R model Eq. (1)) is solved by the $4^{\text {th }}$ order Runge-Kutta method (Step D) to give the segregation profile, i.e., the axial concentration profile of the jetsam $\left(C_{a v e}\right.$ vs. $z$ ). The initial values of the bulk and wake jetsam fractions $\left(C_{B 0}\right.$ and $\left.C_{W 0}\right)$ at the bottom boundary $(z=0)$ are set to $C_{i t}$ (Standard G-R model calculation), while the initial value of the bulk phase gradient $\left(\partial C_{B} / \partial z=0 @ z=0\right)$ is $C_{B 0}\left(C_{B 0}-1\right) / \beta$ (Step C).

The problem of this set of coefficients is that it always over/under-predicts the total mass fraction of the jetsam in the bed, and this contradicts the mass conservation as shown in the second subfigure of Fig. 3. The new 


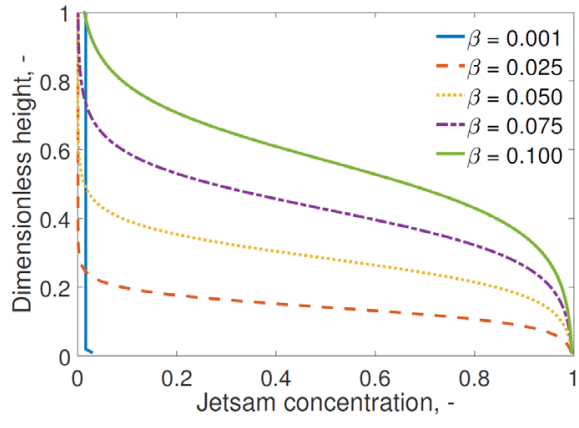

(a)

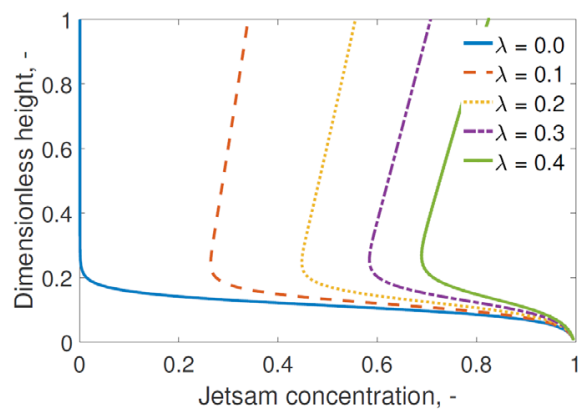

(b)

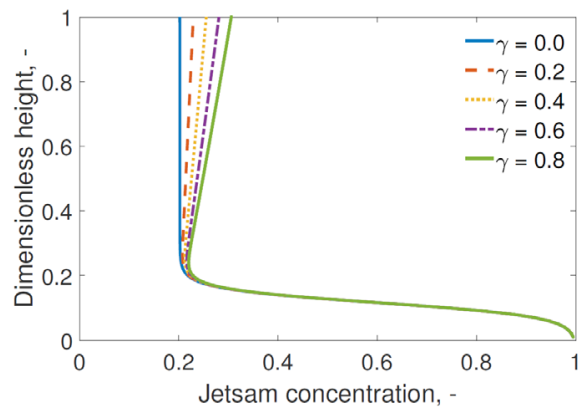

(c)

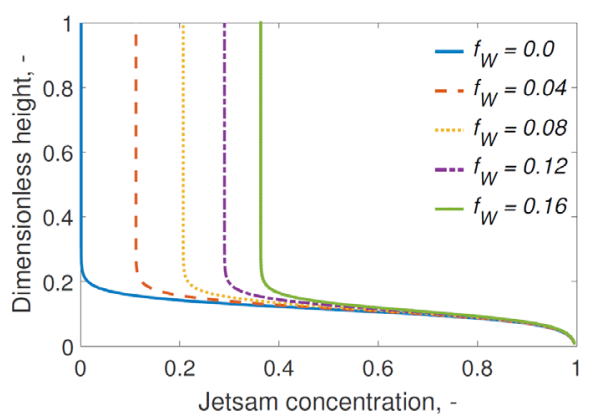

(d)

Fig. 2 Effect of the G-R model coefficients' values on the predicted jetsam profile in a binary-mixture fluidized bed

conservative model calculation is shown in Fig. 4. In this procedure, we generate multiple solutions (Step C-E) by factorizing the set of coefficients (Step H0). Then, we filter them (Step F-G) to choose the correct one (Step H1), which satisfies the mass conservation (i.e., the area under the curve $=$ total jetsam fraction), as shown by Fig. 4 .
Table 1 A set of the most cited equations in the literature for the G-R model coefficients

$$
\begin{gathered}
f_{W}=\frac{1}{2}-\frac{9}{16} \cos \left(\frac{\theta_{W}}{2}\right)+\frac{1}{16} \cos \left(\frac{3 \theta_{W}}{2}\right) \\
\theta_{W}=\left\{\begin{array}{cc}
0 & d_{B}<0.01 \\
70\left(100 d_{B}-1\right) & 0.01<d_{B}<0.03 \\
140 & 0.03<d_{B}
\end{array}\right.
\end{gathered}
$$$$
d_{B}=\left[-\Gamma_{m}+\left(\Gamma_{m}^{2}+\frac{4 d_{b m}}{d_{t}}\right)^{0.5}\right]^{2} \frac{d_{t}}{4}
$$$$
\Gamma_{m}=\frac{2.56 \times 10^{-2}}{u_{m f}}\left[\frac{d_{t}}{g}\right]^{0.5}
$$

$d_{b m}=2.59 g^{-0.2}\left[\left(u_{0}-u_{m f}\right) A_{t}\right]^{0.4}$

$$
\begin{gathered}
\beta=\frac{4}{9 H^{2}} \frac{\alpha^{2} d_{B}^{2}\left(1-\delta_{B}^{\prime}\right)}{1-\left(1-f_{W}\right) \delta_{B}^{\prime}} \\
\lambda=\frac{4}{3} \frac{f_{W}}{0.6\left(\frac{\rho_{j}}{\rho_{f}}\right)\left(\frac{d_{j}}{d_{f}}\right)^{\frac{1}{3}}}
\end{gathered}
$$

$\gamma=\frac{3}{2} \frac{u_{m f}}{u_{B}} \frac{H}{d_{B} \varepsilon_{m f}}$

where the bubble rising velocity $u_{B}$ and the bubble volume fraction in the bed $\delta_{B}^{\prime}$ are given by:

$u_{B}=u_{0}-u_{m f}+0.711 \sqrt{g d_{B}}$

$\delta_{B}^{\prime}=\frac{u_{0}-u_{m f}}{\left(1-f_{W}\right) u_{B}}$ and $\alpha=\frac{u_{B} \varepsilon_{m f}}{u_{m f}}$

This method can then give a much more realistic prediction based on mass conservation.

The criteria used to vary the correction coefficients are based on the variation of the predicted jetsam profile, as shown in Fig. 2. We made iteration loops of each correction coefficient of limits between 0 and 1 . We increased the upper limit to 5000, only in a few cases when the mass conservation did not achieve within the major range. For example, in few cases, the value of $\beta$ coefficient, obtained from the bubble-based correlations is very small. Thus, a sufficient magnification is required to reach the mass conservation criteria. Also, to reduce the computation time, we set $\gamma$ coefficient to -1 , because this coefficient varies slightly, as shown in Fig. 2.

The proposed numerical solution uses the initial boundary value problem setup, in which the differentiated variables should be assigned to the initial value at zero-boundary $(@ z=0)$. Fig.5 shows the effect of the initial bulk concentration on the segregation profile. Fig. 5 shows that 


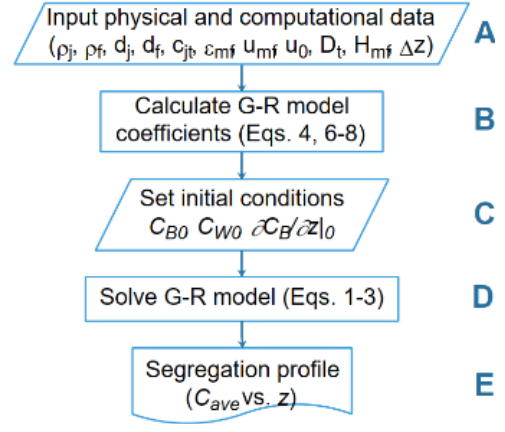

(a)

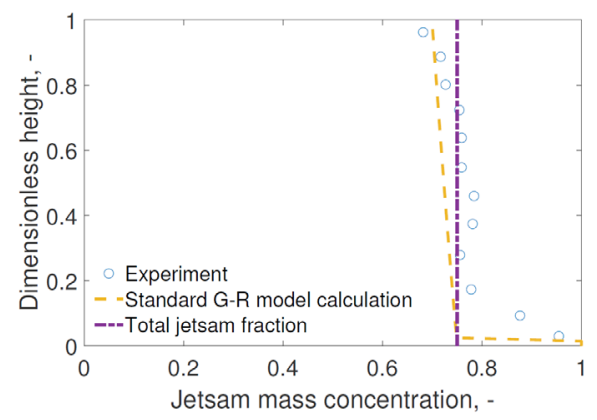

(b)

Fig. 3 (a) Flowchart of the standard G-R model calculation;

(b) Violation of the mass conservation of the jetsam concentration profile predicted by the standard G-R model calculation

assigning the initial bulk concentration to the total jetsam fraction results in non-realistic segregation profile. Thus, we chose the maximum value of $99 \%$ of segregation as the initial jetsam concentration. Although mathematically, this value is arbitrary and can be assigned to any value, we set it to the physically maximum value for segregating systems. However, for the mixing systems, we applied a fictitious initial-boundary below zero-level $(z=0)$, and as a result, the segregation profile starts at a value between unity and the total jetsam fraction. We did not assign a unity initial for the bulk jetsam fraction to avoid a numerical singularity.

\section{Results}

To stand on the present model validity, we compared its simulation results with different experimental data available in the literature of different binary-mixture systems, e.g., density-segregating systems (two materials of same particles' size), size-segregating systems (same material of two particles' sizes), and complex-segregating systems (two materials and two particles' sizes). Also, we compared the present model results with CFD model predictions to demonstrate the present model feasibility over those commonly-used models.

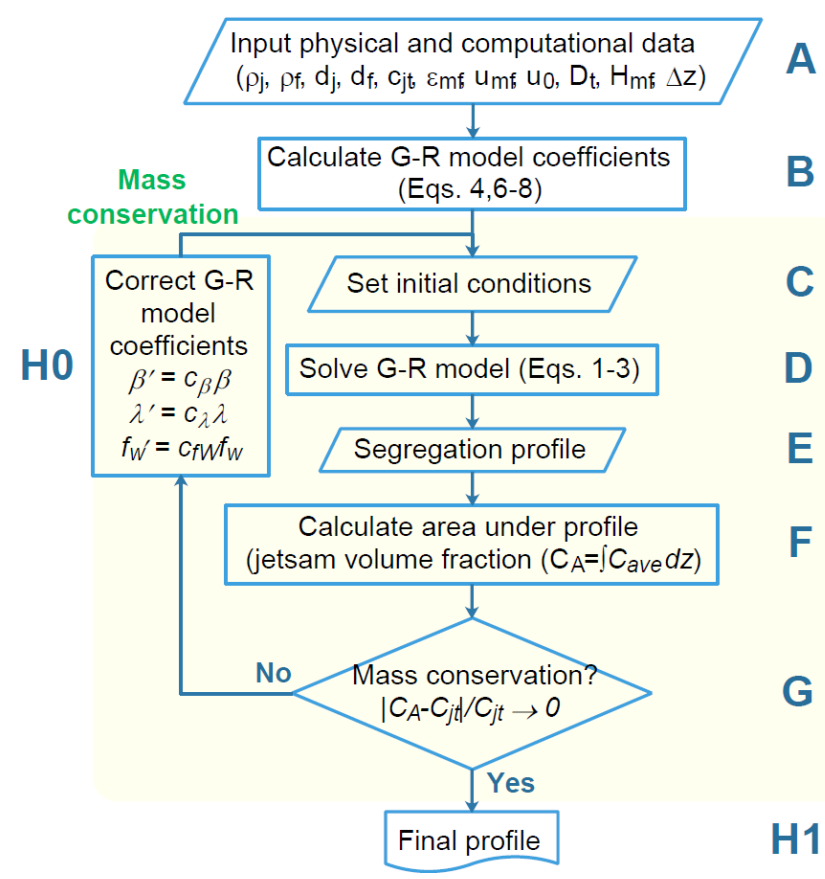

(a)

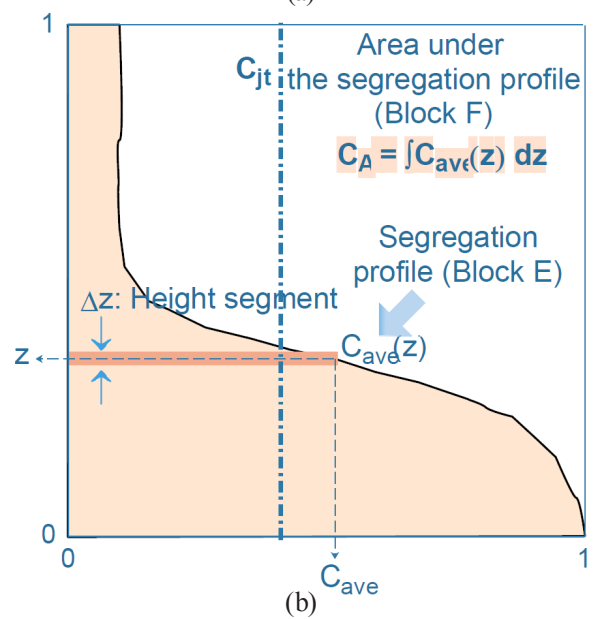

Fig. 4 (a) Flowchart of the conservative G-R model calculation; (b) Estimation of the predicted total jetsam mass fraction from a given jetsam concentration profile.

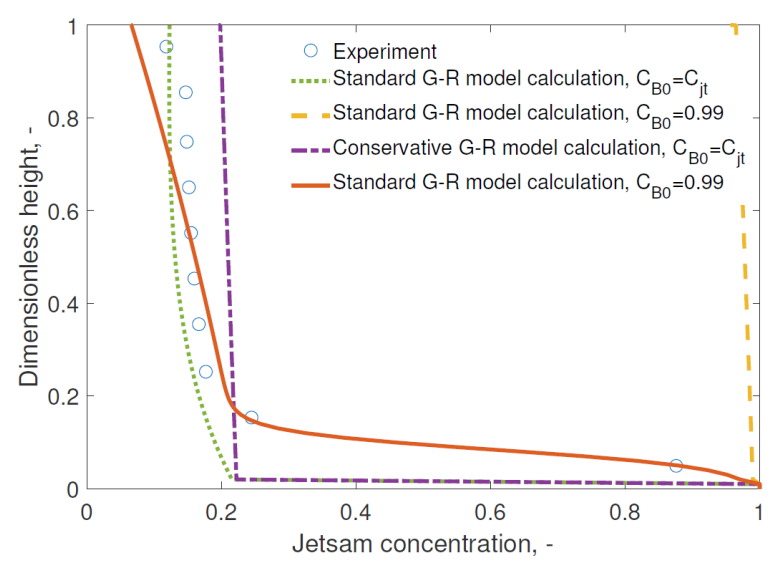

Fig. 5 Effect of initial bulk concentration on the segregation profile. 


\subsection{Segregating fluidized beds}

\subsubsection{Density-segregating systems}

The density-segregating fluidized beds exist in many industrial applications, such as fuel mixing in fluidized bed combustors. If the system contains mono-size particles, the drag force will be equal. In this case, the separation of particles happens when the inertia force is comparable to the drag force. The present conservative G-R model uses the binary-mixture system properties to estimate the bubble-based set of coefficients. After that, iterative correction is carried out to satisfy the mass conservation. We selected experimental cases available in the literature of different density-segregating systems, which represent different mixing/segregation patterns (see Table 2) [12, 23]. For example, in Fig. 6, systems G3750P3750 and G231P231 (upper row in Fig. 6) shows partial segregation cases, while systems A3750P3750 and G3750A3750 (lower row in Fig. 6) represent strong segregation and mixing conditions, respectively. Furthermore,

Table 2 Properties of experimental cases of density-segregating systems available in the literature

\begin{tabular}{lcccc}
\hline System ID & $\rho j / \rho f$ & $d j / d f$ & $u / u_{m f}$ & Ref. \\
\hline G3750P3750 & $2200 / 920$ & $3750 / 3750$ & $1.420 / 1.153$ & {$[12]$} \\
A3750P3750 & $1400 / 920$ & $3750 / 3750$ & $1.300 / 1.272$ & {$[12]$} \\
G3750A3750 & $2200 / 1400$ & $3750 / 3750$ & $1.680 / 1.605$ & {$[12]$} \\
G231P231 & $2476 / 1064$ & $231 / 231$ & $0.076 / 0.042$ & {$[23]$} \\
\hline
\end{tabular}
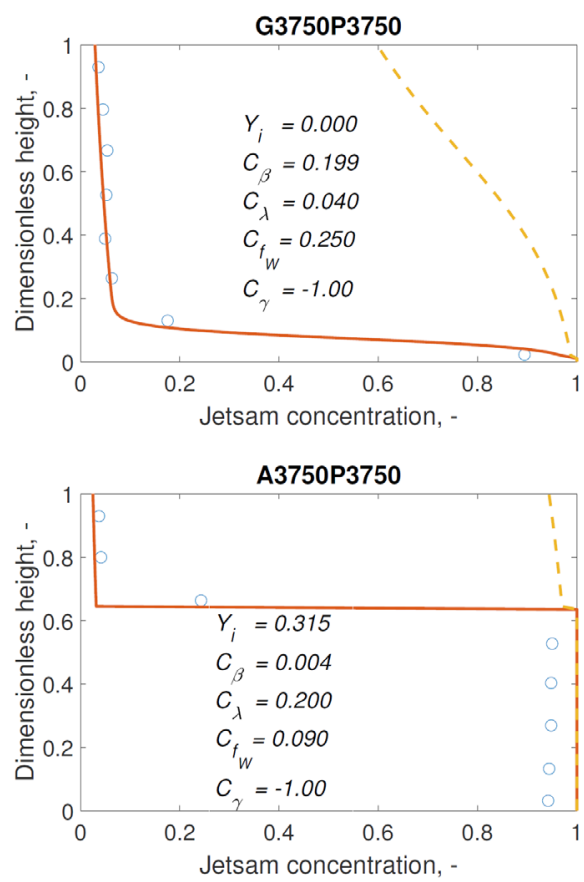

Fig. 6 shows good agreement of the conservative G-R model with the experimental data and poor prediction in the case of the standard G-R model.

\subsubsection{Size-segregating systems}

In mixture of mono-density, binary-size, the drag and inertia forces are unequal for all particles. However, the difference in the drag-inertia balance is not significant near the minimum fluidization velocity of the mixture. In this case, strong segregation does not occur.

We surveyed the literature for size-segregating experimental cases for verification of the present model (see Table 3) [23-25]. The validation results of different mixing patterns are shown in Fig. 7. Also, Fig. 7 demonstrates the good agreement of the present G-R model with the experimental data of size-segregating systems. Moreover, there were no strong segregation cases found, but only weak segregation cases appeared in systems G500S125 and G555G281 (upper row in Fig. 7). While the other two systems G231G116I and G231G116II (lower row in Fig. 7) showed strong mixing patterns.

\subsubsection{Complex-segregating systems}

In real applications, there is no ideal homogeneity in fluidized bed systems, i.e., there is a difference in both density and size of particles. For further verification of the
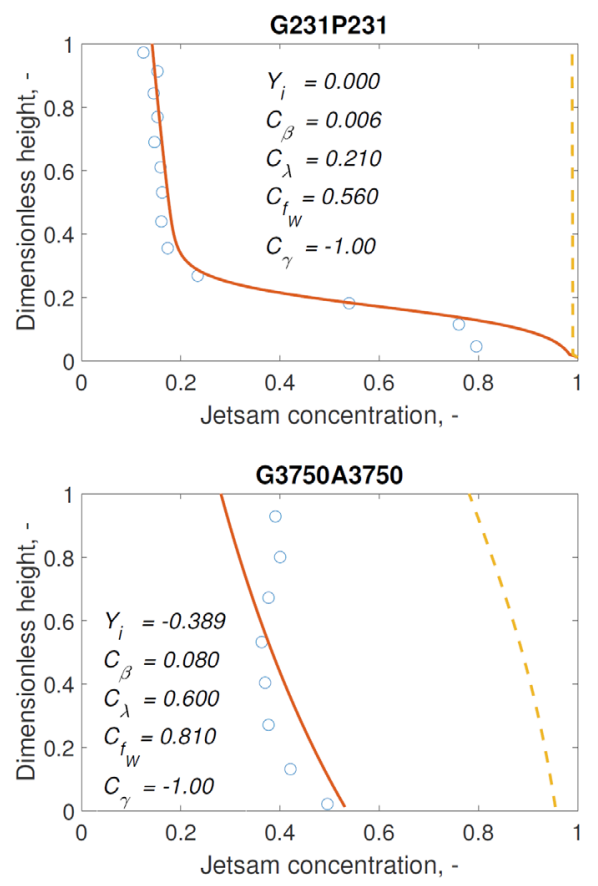

Fig. 6 Simulation results of density-segregating systems. (Blue circle: Experimental from the literature; Yellow dashed line: Standard G-R model calculation; Red solid line: Conservative G-R model calculation of the current work). The titles of subfiguresrefer to the system IDs, as stated in

Table 2. According to them, the upper row shows partial segregation cases, while the lower row shows strong and weak segregation cases. 
Table 3 Properties of experimental cases of size-segregating systems available in the literature

\begin{tabular}{lcccc}
\hline System ID & $\rho j / \rho f$ & $d j / d f$ & $u / u_{m f}$ & Ref. \\
\hline G500S125 & $2540 / 2600$ & $500 / 125$ & $0.080 / 0.046$ & {$[24]$} \\
G555G281 & $2490 / 2490$ & $555 / 281$ & $0.179 / 0.151$ & {$[25]$} \\
G231G116I & $2476 / 2476$ & $231 / 116$ & $0.057 / 0.022$ & {$[23]$} \\
G231G116II & $2476 / 2476$ & $231 / 116$ & $0.076 / 0.043$ & {$[23]$} \\
\hline
\end{tabular}

present model, we investigated several cases from the literature that represent this complex structure (see Table 4). Fig. 8 shows prediction comparisons between the current conservative G-R model and the standard G-R model of those complex binary-mixture systems. The standard G-R model failed to give the partial segregation trend of the experimental results. Also, the standard G-R model violated the mass conservation. On the other hand, the present conservative model showed good predictions. The reduction of bubble-based parameters reflects the role of bubbles on segregation phenomenon. The original theory of Gibilaro and Rowe assumes that the bubbles cause axial displacement of solids. Some studies neglected the axial dispersion mechanism [17, 19, 26]. García-Ochoa et al. [12] reported that this axial dispersion should not be neglected. The bubble mixing influence was experimentally approved by Park and Choi [27]. However, Formisani et al. [28-30] reported independent segregation from excess gas velocity (bubbles) in the transition zone between the initial and final fluidization velocities. It is clear that the difference of minimum fluidization velocities significantly influences the segregation. For example, in density segregating systems, there will be one component in the bubbling region (flotsam) and the other component are not fluidized even (jetsam). Thus, the bubbles mixing mechanism will be smaller and even negligible when the difference in minimum fluidization velocities is higher e.g., Geldart A-B or B-D mixtures. It is worth mentioning here that in all of the predicted results, the initial height (segregation layer thickness) was optimized to give the best fit with the experimental data. And a general correlation for this parameter need to be fitted with the fluidization ratio of the mixture.

\subsection{Effect of the segregation layer height}

The bubbling action inside the fluidized bed depends primarily on the fluidization number (i.e., $\left.u_{0} / u_{m f}\right)$. Meanwhile, the minimum fluidization depends on the bed system composition [31]. Therefore, any modeling of a real phenomenon in the fluidization process, such as segregation, should take into account this critical parameter. The G-R model coefficients were already estimated based on those parameters, and similarly should be the correction of the model. Based on this, we optimized the segregation layer height $\left(Y_{i}\right)$ and inputted it as an initial-boundary value in all the previously shown segregation profiles.
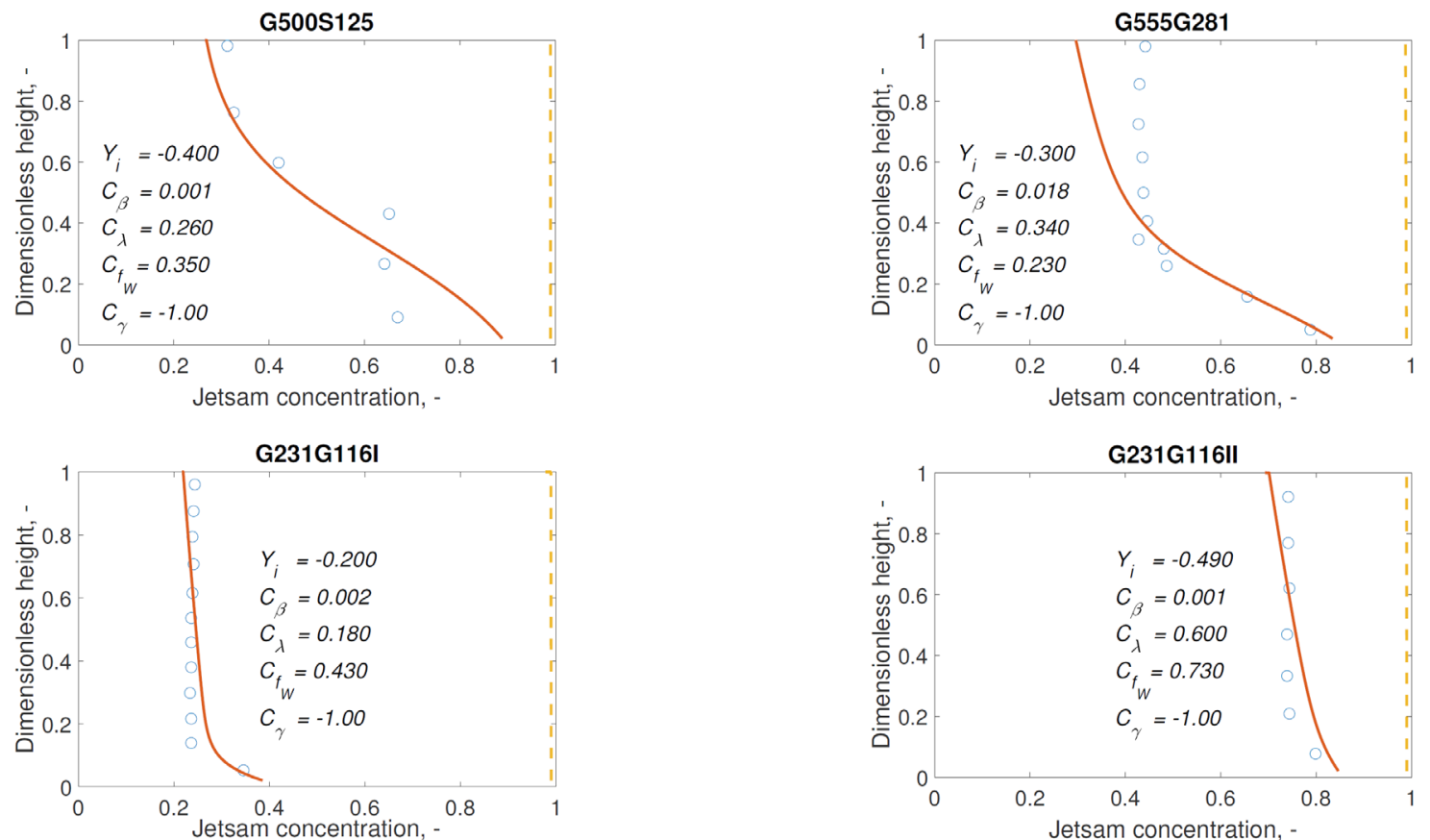

Fig. 7 Simulation results of size-segregating systems. (Blue circle: Experimental from the literature; Yellow dashed line: Standard G-R model calculation; Red solid line: Conservative G-R model calculation of the current work). The titles of subfigures refer to the system IDs, as stated in Table 2. According to them, the upper row shows partial segregation cases, while the lower row shows strong and weak segregation cases. 
Table 4 Properties of experimental cases of complex-segregating systems available in the literature

\begin{tabular}{lcccc}
\hline System ID & $\rho j / \rho f$ & $d j / d f$ & $u / u_{m f}$ & Ref. \\
\hline L112G281 & $11320 / 2490$ & $112 / 281$ & $0.119 / 0.105$ & {$[25]$} \\
C235G565I & $8750 / 2510$ & $235 / 565$ & $0.290 / 0.258$ & {$[26]$} \\
C235G565II & $8750 / 2510$ & $235 / 565$ & $0.330 / 0.258$ & {$[26]$} \\
G116P275 & $2476 / 1064$ & $116 / 275$ & $0.076 / 0.038$ & {$[23]$} \\
\hline
\end{tabular}

The segregation layer thickness $\left(Y_{i}\right)$ is an indication of the strength of segregation, and it is defined as the height of the bottom layer containing $100 \%$ jetsam.

Because there is no accurate expression in the literature for this parameter, we proposed here approximation of it. The proposed estimate determines the segregation layer height as a function of the fluidization number. Here, the minimum fluidization velocity of the system can express the binary-mixture system properties such as densities, sizes, and total mass ratio. Thus, in Fig. 9, we assumed that the fluidization ratio could then represent the segregation pattern (i.e., strong segregation, partial segregation, or mixing). However, in the mixing cases (i.e., no segregation), the segregation layer thickness parameter is more mathematical than physical. A general correlation can be formulated from the optimized segregation layer thickness (see Fig. 9) in Eq. (5):

$$
\begin{aligned}
& \frac{Y_{i}}{C_{j t}} \\
& =\left\{\begin{array}{cc}
15.589\left(\frac{u_{0}}{u_{m f}}\right)^{3.76} \exp \left[-3.275\left(\frac{u_{0}}{u_{m f}}\right)^{4.76}\right], & \text { for } u_{0} \leq u_{m f, j} \\
-0.8, & \text { for } u_{0}>u_{m f, j}
\end{array}\right.
\end{aligned}
$$

where $u_{m f}$ is the minimum fluidization of the mixture calculated from minimum fluidization velocities of flotsam and jetsam as proposed Hoffman et al. [26] and Cheung et al. [32], Eq. (6):

$u_{m f}=u_{m f, f}\left(\frac{u_{m f, j}}{u_{m f, f}}\right)^{x_{j}^{2}}$.

\subsection{Comparison with the other models}

\subsubsection{Comparison with the recent macroscopic models}

Recently, macroscopic models for binary-mixture fluidized bed were published $[8,13]$. These models were verified by optimizing a fitting parameter, which gives a good match with the experimental data of binary-mixture systems (see Table 5). Fig. 10 presents a comparison among the predictions of the axial jetsam concentrations by using these models and the present model. The current model used the segregation
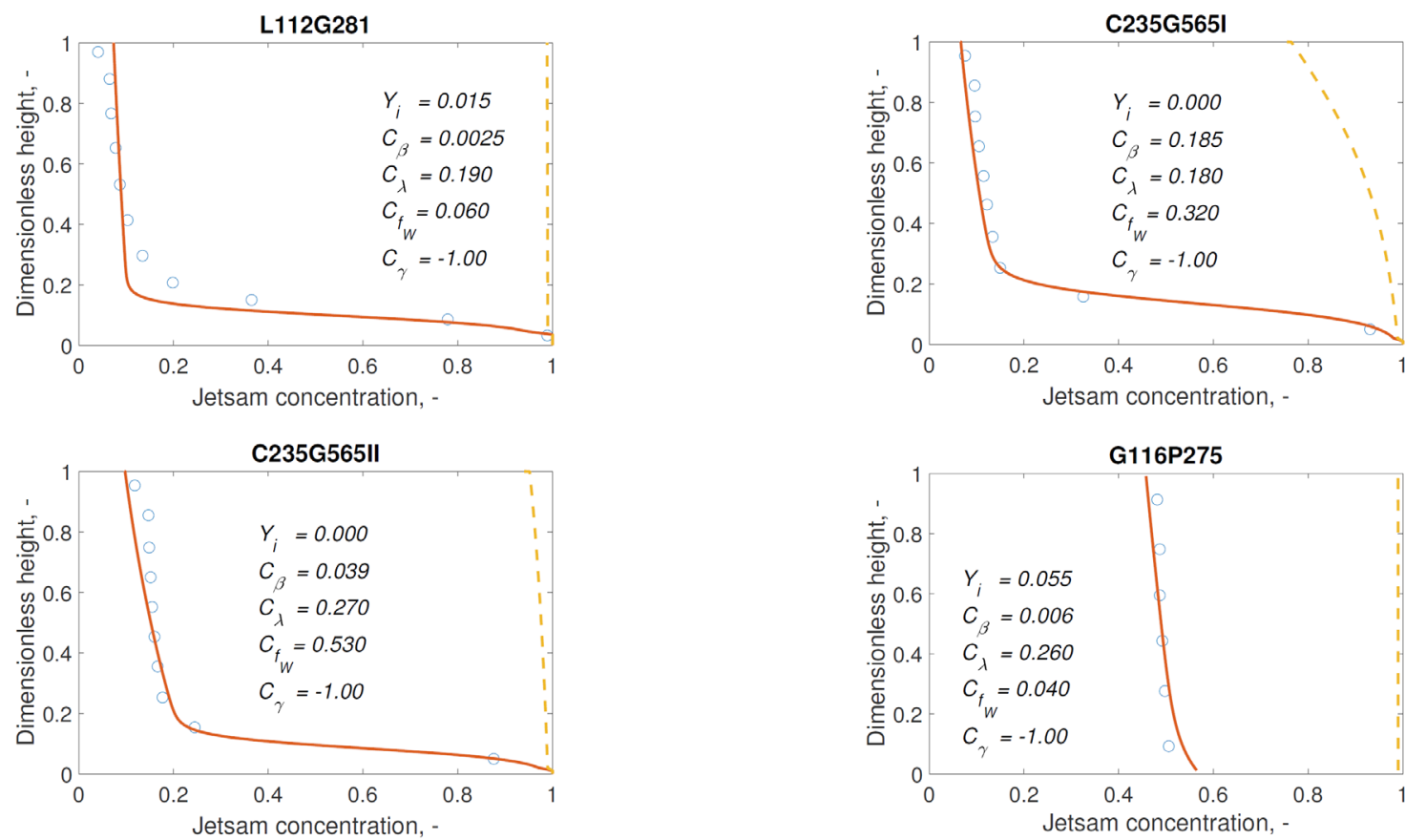

Fig. 8 Simulation results of complex-segregating systems. (Blue circle: Experimental from the literature; Yellow dashed line: Standard G-R model calculation; Red solid line: Conservative G-R model calculation of the current work). The titles of subfigures refer to the system IDs, as stated in Table 4. According to them, the first three subfigures show partial segregation cases, while the last subfigure shows a strong mixing case. 


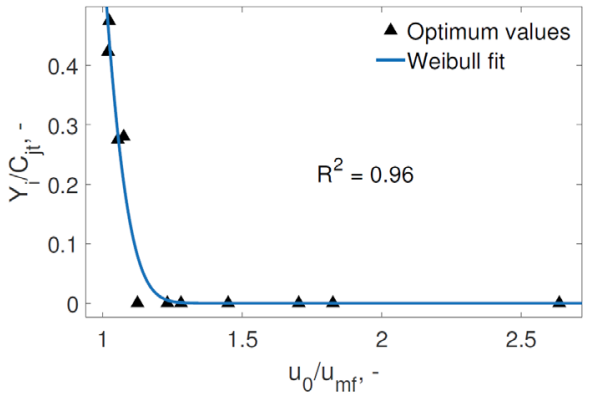

Fig. 9 Effect of fluidization velocity on the segregation layer thickness.

Table 5 Properties of binary-mixture systems available in the literature used in the fitting of the recently published macroscopic models

\begin{tabular}{lcccc}
\hline System ID & $\rho j / \rho f$ & $d j / d f$ & $u / u_{m f}$ & Ref. \\
\hline IO225G90I & $4600 / 2500$ & $225 / 90$ & $0.060 / 0.019$ & {$[8]$} \\
IO225G90II & $4600 / 2500$ & $225 / 90$ & $0.120 / 0.103$ & {$[8]$} \\
IO378G90 & $4600 / 2500$ & $378 / 90$ & $0.180 / 0.108$ & {$[8]$} \\
B268G535 & $8670 / 2480$ & $268 / 535$ & $0.384 / 0.337$ & {$[13]$} \\
\hline
\end{tabular}

layer thickness correlation Eq. (4); however, the other models were optimized by a fitting parameter. In general, the present model showed better agreement with the experimental data compared to these published macroscopic models.

\subsubsection{Comparison with the CFD model}

In model assessment studies, comparison with the commonly-used model is important to stand on the proposed model feasibility.
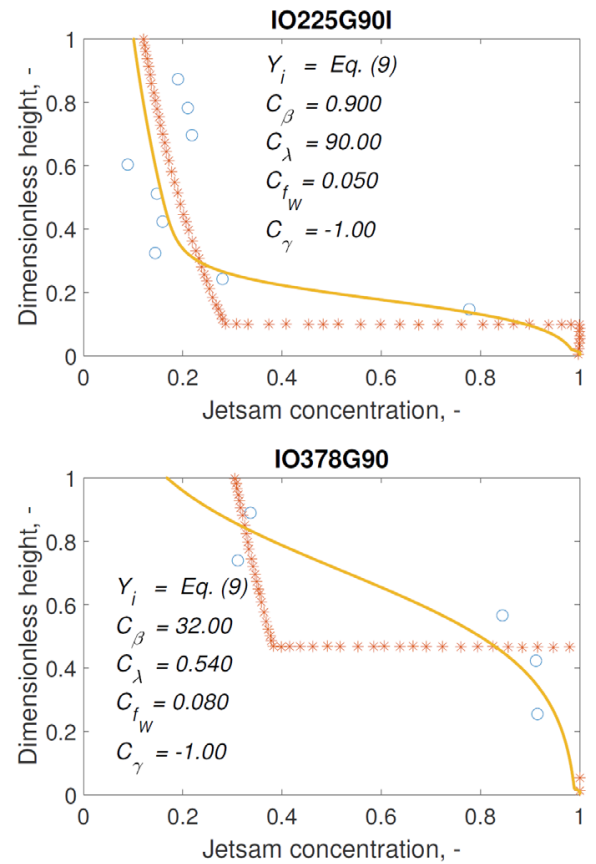

In this study, we compare the present conservative G-R model predictions with that of the Two-Fluid Model (TFM). The TFM is the most commonly used CFD model in the literature. This model assumes both the gas and solid phases as continua. The governing equations are found in the theory guide of the commercial code ANSYS FLUENT (student license). The settings of the CFD model were applied, as recommended in the published literature (see Table 6) [33-35]. For example, the gas-solid drag closure is the most significant element in the CFD model, and the Gidaspow model is the most verified model. The other parameters are selected based on the literature's best-practice knowledge. For the present comparison, we chose binary-mixture cases available in the literature, which represent different segregation patterns, as given in Table 7. The TFM-CFD solution predicts the low jetsam composition profiles pretty well, except the bottom region as shown in Fig. 11. However, mass conservation is underpredicted. This can be attributed to the bed expansion because the results were calculated on the fluidized condition with normalizing by the fluidized bed height. This is the major method applied in the literature, and there is a shortcoming of the FLUENT code when freezing the bed, the results change to perfect mixing condition. Conversely, the present conservative model gives better agreement with the experimental data as compared to that obtained from the CFD-TFM. Moreover, the
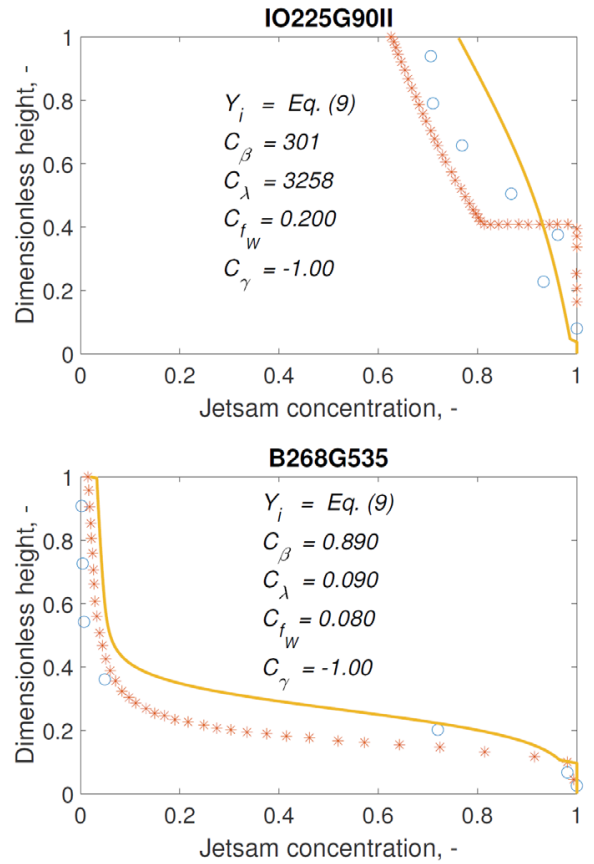

Fig. 10 Comparison between the predictions of the present conservative G-R model and the previous macroscopic models. (Blue circle: Experimental data from the literature; Red star: Previous macroscopic models; Yellow dashed line: Standard G-R model calculation; Red solid line: Conservative G-R model calculation of the current work). The titles of subfigures refer to the system IDs, as stated in Table 5. According to them, all the subfigures 
Table 6 Summary of the CFD model settings

\begin{tabular}{lccc}
\hline Gas-solid drag & {$[33]$} & $\begin{array}{c}\text { Particle-wall restitution } \\
\text { coefficient }\end{array}$ & 0.90 \\
\hline Solid-solid drag & {$[34]$} & Specularity coefficient & 0.60 \\
Viscosity closure & $\begin{array}{c}\text { KTGF* } \\
{[35]}\end{array}$ & Maximum packing & 0.63 \\
$\begin{array}{l}\text { Particle-particle } \\
\text { restitution coefficient }\end{array}$ & 0.90 & Time step & 0.001 \\
\hline
\end{tabular}

* Kinetic Theory of Granular Flow

Table 7 Properties of experimental cases available in the literature for a validation comparison with the CFD model

\begin{tabular}{lcccc}
\hline System ID & $\rho_{j} / \rho_{f}$ & $d_{j} / d_{f}$ & $u / u_{m f}$ & Ref. \\
\hline C273G461I & $8860 / 2950$ & $273 / 461$ & $0.337 / 0.314$ & {$[17]$} \\
C273G461II & $8860 / 2950$ & $273 / 461$ & $0.336 / 0.319$ & {$[17]$} \\
C273G461III & $8860 / 2950$ & $273 / 461$ & $0.650 / 0.319$ & {$[17]$} \\
C273G461IV & $8860 / 2950$ & $273 / 461$ & $0.337 / 0.331$ & {$[17]$} \\
\hline
\end{tabular}

current model consumes significantly less computational time as compared to the CFD-TFM.

\section{Conclusion}

The presented conservative macroscopic model for binary-mixture fluidized beds takes into account all the mechanisms responsible for mixing and segregation as proposed
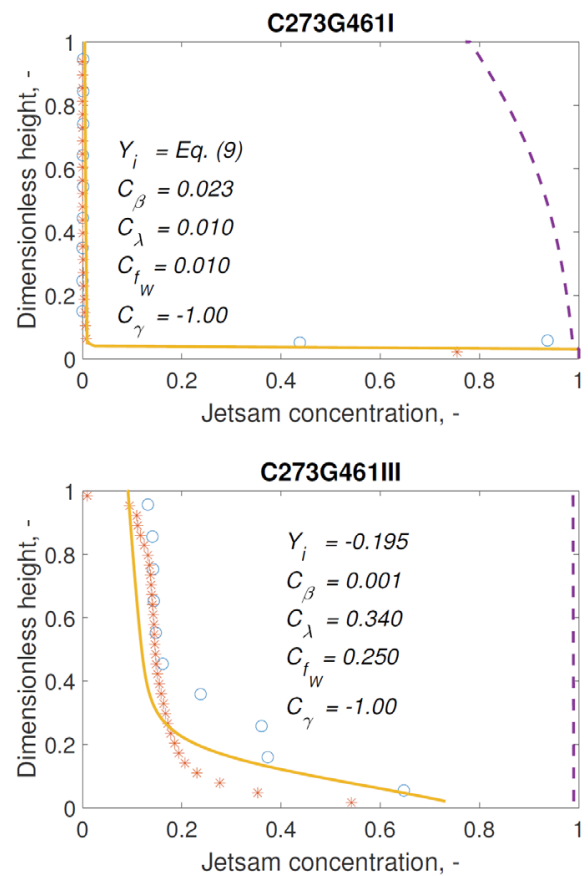

by Gibilaro and Rowe [6]. A full numerical solution method is applied. Moreover, the proposed model corrects the prediction of the jetsam profile by the mass conservation and modification of the model coefficients. The limits of the correction factors are 0 and 1, except for few cases where the mass conservation are not achieved. A validation test is carried out using experimental data available in the literature of diverse binary-mixture systems containing solids of different densities and sizes. The conservative model shows a good prediction of jetsam profile in all binary-mixture beds. Also, the comparison with the CFD model reveals that the present macroscopic model is of higher reliability and accuracy than the CFD model. Finally, a correlation for the segregation layer height is proposed as a function of the fluidization velocity ratio.

\section{Acknowledgments}

The research reported in this paper has been supported by the National Research, Development, and Innovation Fund (TUDFO/51757/2019-ITM, Thematic Excellence Program and the National Research, Development, and Innovation Fund of Hungary in the frame of FIEK'16-1-2016-0007 (Higher Education and Industrial Cooperation Center).
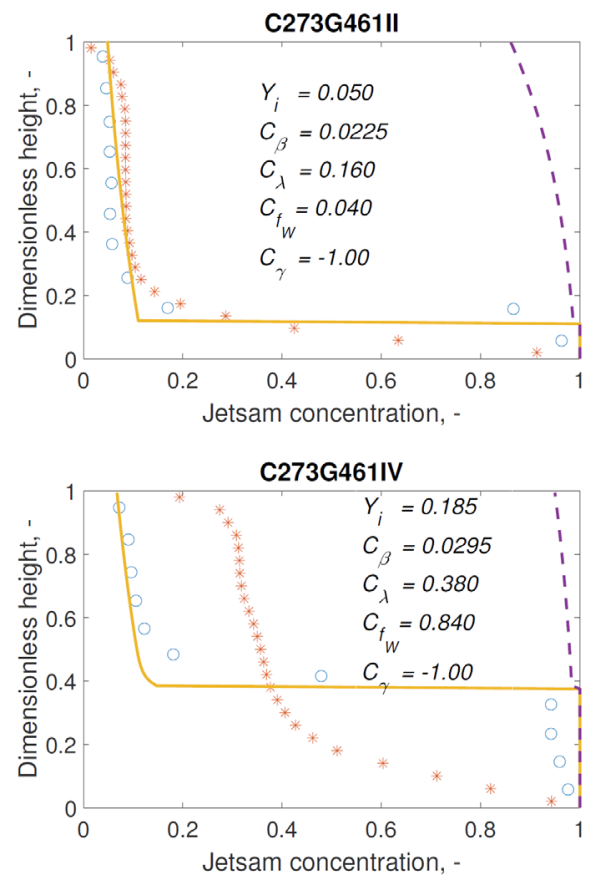

Fig. 11 Comparison between the predictions of the present conservative G-R model and the CFD model. (Blue circle: Experimental data from the literature; Red star: CFD model; Yellow dashed line: Standard G-R model calculation; Red solid line: Conservative G-R model calculation of the current work). The titles of subfigures refer to the system IDs, as stated in Table 6 . According to them, the first three subfigures show strong segregation cases, while the bottom right subfigure shows a weak segregation case. 


\section{Nomenclature}

$\alpha \quad$ numerical coefficient

$\beta \quad$ axial mixing/segregation coefficient

$\theta \quad$ angle

C volumetric concentration

$\delta_{B}^{\prime} \quad$ volume fraction of bubble involving wake

$d_{B} \quad$ bubble diameter $\mathrm{m}$

$d_{B m} \quad$ maximum bubble diameter $\mathrm{m}$

$D_{t} \quad$ bed diameter m

d particle diameter $\mathrm{m}$

$f_{W} \quad$ solids volume fraction in the wake phase -

$g$ gravitational acceleration $\mathrm{m} / \mathrm{s}^{2}$

$\gamma \quad$ phase exchange/circulation coefficient

$H \quad$ bed height

$\lambda$ circulation/segregation coefficient

$P \quad$ pressure

$u \quad$ velocity

$x \quad$ mass concentration
Subscripts

$0 \quad$ superficial velocity

ave average

$b$ bed

$B \quad$ bulk phase, bubble

$f \quad$ flotsam

g gas

$j \quad$ jetsam

jt total jetsam mf minimum fluidization

$m f \quad$ minimum fluidization

$s \quad$ solids, small

$W \quad$ wake phase

Abbreviations

CFD Computational Fluid Dynamics

G-R Gibilaro and Rowe

KTGF Kinetic Theory of Granular Flow

TFM Two-Fluid Model

TPT Two-Phase Theory

\section{References}

[1] Chladek, J., Jayarathna, C. K., Moldestad, B. M. E., Tokheim, L-A. "Fluidized bed classification of particles of different size and density", Chemical Engineering Science, 177, pp. 151-162, 2018. https://doi.org/10.1016/j.ces.2017.11.042

[2] Zhou, Q., Wang, J. "CFD study of mixing and segregation in CFB risers: Extension of EMMS drag model to binary gas-solid flow", Chemical Engineering Science, 122, pp. 637-651, 2015. https://doi.org/10.1016/j.ces.2014.10.025

[3] Ahmad, N., Tong, Y., Lu, B., Wang, W. "Extending the EMMSbubbling model to fluidization of binary particle mixture: Parameter analysis and model validation", Chemical Engineering Science, 200, pp. 257-267, 2019. https://doi.org/10.1016/j.ces.2019.02.016

[4] Lu, L., Xu, Y., Li, T., Benyahia, S. "Assessment of different coarse graining strategies to simulate polydisperse gas-solids flow", Chemical Engineering Science, 179, pp. 53-63, 2018. https://doi.org/10.1016/j.ces.2018.01.003

[5] Qin, Z., Zhou, Q., Wang, J. "An EMMS drag model for coarse grid simulation of polydisperse gas-solid flow in circulating fluidized bed risers", Chemical Engineering Science, 207, pp. 358-378, 2019. https://doi.org/10.1016/j.ces.2019.06.037

[6] Gibilaro, L. G., Rowe, P. N. "A model for a segregating gas fluidised bed", Chemical Engineering Science, 29(6), pp. 1403-1412, 1974. https://doi.org/10.1016/0009-2509(74)80164-8

[7] Al-Agha, M. S., Szentannai, P. "One-dimensional numerical model for prediction of jetsam concentration in segregating fluidized beds", Thermal Science, 23(2), pp. 1173-1187, 2019. https://doi.org/10.2298/TSCI170418066A

[8] Turrado, S., Fernández, J. R., Abanades, J. C. "Investigation of the Segregation of Binary Mixtures with Iron-Based Particles in a Bubbling Fluidized Bed", ACS Omega, 4(5), pp. 9065-9073, 2019. https://oi.org/10.1021/acsomega.9b00674

[9] Al-Agha, M. S., Szentannai, P. "An Efficient Model for Predicting the Segregation Profile of Binary Fluidized Beds", Periodica Polytechnica Chemical Engineering, 63(1), pp. 147-159, 2019. https://doi.org/10.3311/PPch.11997

[10] Fotovat, F., Chaouki, J., Bergthorson, J. "Distribution of large biomass particles in a sand-biomass fluidized bed: Experiments and modeling", AIChE Journal, 60(3), pp. 869-880, 2014. https://doi.org/10.1002/aic.14337

[11] Leaper, M. C., King, A. C., Burbidge, A. S. "Total Solution of the Gibilaro and Rowe Model for a Segregating Fluidized Bed", Chemical Engineering \& Technology, 30(2), pp. 161-167, 2007. https://doi.org/10.1002/ceat.200600139

[12] García-Ochoa, F., Romero, A., Villar, J. C., Bello, A. "A study of segregation in a gas-solid fluidized bed: Particles of different density", Powder Technology, 58(3), pp. 169-174, 1989. https://doi.org/10.1016/0032-5910(89)80111-1

[13] Girimonte, R., Formisani, B., Vivacqua, V. "The concentration profile of two-solid beds after slow defluidization: Model and experiment", Chemical Engineering Journal, 359, pp. 1006-1012, 2019. https://doi.org/10.1016/j.cej.2018.11.084

[14] Girimonte, R., Formisani, B., Vivacqua, V. "The relationship between fluidization velocity and segregation in two-component gas fluidized beds: Density- or size-segregating mixtures", Chemical Engineering Journal, 335, pp. 63-73, 2018. https://doi.org/10.1016/j.cej.2017.10.135

[15] Toomey, R. D., Johnstone, H. F. "Gaseous fluidization of solid particles", Chemical Engineering Progress, 48, pp. 220-226, 1952.

[16] Rowe, P. N., Nienow, A. W. "Particle mixing and segregation in gas fluidised beds. A review", Powder Technology, 15(2), pp. 141-147, 1976. https://oi.org/10.1016/0032-5910(76)80042-3 
[17] Naimer, N. S., Chiba, T., Nienow, A. W. "Parameter estimation for a solids mixing $\mid$ segregation model for gas fluidised beds", Chemical Engineering Science, 37(7), pp. 1047-1057, 1982. https://doi.org/10.1016/0009-2509(82)80135-8

[18] Chiba, T., Kobayashi, H. "Solid exchange between the bubble wake and the emulsion phase in a gas-fluidised bed", Journal of Chemical Engineering of Japan, 10(3), pp. 206-210, 1977. https://doi.org/10.1252/jcej.10.206

[19] Tanimoto, H., Chiba, S., Chiba, T., Kobayashi, H. "Jetsam descent induced by a single bubble passage in three-dimensional gas-fluidized beds", Journal of Chemical Engineering of Japan, 14(4), pp. 273-276, 1981. https://oi.org/10.1252/jcej.14.273

[20] Horio, M., Nonaka, A. "A generalized bubble diameter correlation for gas-solid fluidized beds", AIChE Journal, 33(11), pp. 1865-1872, 1987. https://doi.org/10.1002/aic.690331113

[21] Geldart, D. "The effect of particle size and size distribution on the behaviour of gas-fluidised beds", Powder Technology, 6(4), pp. 201-215, 1972. https://doi.org/10.1016/0032-5910(72)83014-6

[22] Kato, K., Wen, C. Y. "Bubble assemblage model for fluidized bed catalytic reactors", Chemical Engineering Science, 24(8), pp. 1351-1369, 1969. https://doi.org/10.1016/0009-2509(69)85055-4

[23] Joseph, G. G., Leboreiro, J., Hrenya, C. M., Stevens, A. R. "Experimental segregation profiles in bubbling gas-fluidized beds", AIChE Journal, 53(11), pp. 2804-2813, 2007. https://doi.org/10.1002/aic.11282

[24] Marzocchella, A., Salatino, P., Di Pastena, V., Lirer, L. "Transient fluidization and segregation of binary mixtures of particles", AIChE Journal, 46(11), pp. 2175-2182, 2000. https://doi.org/10.1002/aic.690461110

[25] Hartholt, G. P. "Particle mixing in gas solid fluidised beds", PhD Thesis, University of Groningen, 1996. [online] Available at: http:// hdl.handle.net/11370/3cec273b-b472-4f04-8d43-a9c11bc69af8 [Accessed: 20 October 2020]

[26] Hoffmann, A. C., Janssen, L. P. B. M., Prins, J. "Particle segregation in fluidised binary mixtures", Chemical Engineering Science, 48(9), pp. 1583-1592, 1993. https://doi.org/10.1016/0009-2509(93)80118-A
[27] Park, H. C., Choi, H. S. "The segregation characteristics of char in a fluidized bed with varying column shapes", Powder Technology, 246, pp. 561-571, 2013. https://doi.org/10.1016/j.powtec.2013.06.019

[28] Formisani, B., Girimonte, R., Longo, T. "The fluidization process of binary mixtures of solids: Development of the approach based on the fluidization velocity interval", Powder Technology, 185(2), pp. $97-108,2008$. https://doi.org/10.1016/j.powtec.2007.10.003

[29] Formisani, B., Girimonte, R., Longo, T. "The fluidization pattern of density-segregating binary mixtures", Chemical Engineering Research and Design, 86(4), pp. 344-348, 2008. https://doi.org/10.1016/j.cherd.2007.11.004

[30] Formisani, B., Girimonte, R., Vivacqua, V. "The interaction between mixture components in the mechanism of binary fluidization", Powder Technology, 266, pp. 228-235, 2014. https://doi.org/10.1016/j.powtec.2014.06.007

[31] Ergun, S. "Fluid Flow Through Packed Columns", Journal of Chemical Engineering Progress, 48(2), pp. 89-94, 1952. [online] Available at: http://dns2.asia.edu.tw/ ysho/YSHOEnglish/2000\%20Engineering/PDF/Che\%20Eng\%20 Pro48,\%2089.pdf [Accessed: 20 October 2020]

[32] Cheung, L., Nienow, A. W., Rowe, P. N. "Minimum fluidization velocity of a binary mixture of different sized particles", Chemical Engineering Science, 29(5), pp. 1301-1303, 1974. https://doi.org/10.1016/0009-2509(74)80137-5

[33] Gidaspow, D. "Multiphase Flow and Fluidization: Continuum and Kinetic Theory Descriptions", Academic Press, Boston, USA, 1994.

[34] Syamlal, M. "The particle-particle drag term in a multiparticle model of fluidization", EG and G Washington Analytical Services Center, Inc., Morgantown, WV, USA, Rep. DOE/MC/213532373, 1987.

[35] Gidaspow, D., Bezburuah, R., Ding, J. "Hydrodynamics of circulating fluidized beds: Kinetic theory approach", presented at $7^{\text {th }}$ International Conference on Fluidization, Gold Coast, Australia, May, 3-8, 1992. [online] Available at: https://www.osti.gov/ biblio/5896246-hydrodynamics-circulating-fluidized-beds-kinetictheory-approach [Accessed: 03 March 2021] 